\title{
A fluorescence temperature-jump study of conformational transitions in myosin subfragment 1
}

\author{
Claus URBANKE* and John WRAY ${ }^{+1}$ \\ *Medizinische Hochschule Hannover, Carl-Neuberg-Str. 1, D-30623 Hannover, Germany, and †Max-Planck-Institut für Medizinische Forschung, Jahnstr. 29, D-69120 \\ Heidelberg, Germany
}

The tryptophan fluorescence of unmodified myosin subfragment 1 (S1) from rabbit and chicken skeletal muscle with various nucleotides and phosphate analogues bound was measured after rapid temperature jumps. The fluorescence decreased during the temperature rise. Under some conditions, this decrease was followed by an increase, reflecting structural transitions within the protein. With adenosine $5^{\prime}$-[ $\beta, \gamma$-imido $]$ triphosphate $(\mathrm{p}[\mathrm{NH}] \mathrm{ppA})$ or with $\mathrm{ADP}$ and $\mathrm{BeF}_{x}$ bound, this rise was very rapid (reciprocal time constant approx. $2000 \mathrm{~s}^{-1}$ ) and varied only slightly with starting temperature, suggesting that, with these ligands, two different protein conformations were present in rapid equilibrium over a large temperature range. In the presence of ATP, the transient included several relaxation processes. Overall, the results suggest that complexes of S1 with ATP or with a number of other ligands exist as a mixture of two forms in temperature-dependent equilibrium. The results throw light on the finding of different forms of S1 in recent crystallographic studies and indicate a surprising lack of strong coupling between myosin's structural state and the nature of the nucleotide bound.

Key words: ATP analogues, muscle contraction, relaxation methods, tryptophan.

\section{INTRODUCTION}

A central goal of research into the mechanism of muscle contraction is to understand the structural basis of the "powerstroke' of the myosin cross-bridge. Such an understanding requires knowledge of the structural transitions that myosin makes while bound to the actin filament. The structural changes in the cross-bridge are thought to be linked to steps in the mechanochemical cycle of hydrolysis of ATP by the actin-myosin complex [1].

Crystallographic studies have led to detailed atomic structural models both for actin and for the head region (subfragment 1, $\mathrm{S} 1$ ) of myosin. The S1 species studied were from several different sources (vertebrate skeletal and smooth muscles, scallop muscle, Dictyostelium) and were crystallized with several different ligands bound, including ADP, adenosine $5^{\prime}$-[ $\beta, \gamma$-imido $]$ triphosphate $(\mathrm{p}[\mathrm{NH}] \mathrm{ppA})$ and the complexes of ADP with fluoride and either aluminium or beryllium. This work has led to the recognition of at least three distinct conformations of $\mathrm{S} 1$ in the absence of actin $[1,2]$. The structure of the complex formed by myosin with actin is not yet known at atomic resolution. However, Holmes [3] has suggested that the transition between two of the described conformations of myosin S1 might constitute part of the conformation change that actin-bound myosin undergoes during the power stroke.

The biochemical kinetics of the interaction between actin, myosin S1 and ATP have been studied in great detail. The hydrolysis of ATP by myosin is often described in terms of Scheme 1, as proposed by Bagshaw and Trentham [4], in which $\mathrm{M}$ represents myosin. Subsequent work has provided evidence for additional steps in this pathway. In this scheme, step 2 is the rapid and almost irreversible formation of a tightly bound complex, step 3 is the rapid and easily reversible cleavage reaction, and steps 4 and 6 are slow under physiological conditions; asterisks indicate increased intensity of tryptophan fluorescence. The kinetics of some steps in the pathway are profoundly modified when myosin is bound to actin. Some myosinnucleotide complexes (M.ATP, M.ADP.P ${ }_{\mathrm{i}}$ ) bind weakly to actin, whereas others (M.ADP) and myosin itself bind strongly: a transition between weak and strong binding is likely to be involved in the power stroke of the myosin cross-bridge [1].

Studies of native tryptophan fluorescence have played a key role in establishing kinetic and other properties of myosin. The binding of ADP or ATP causes an increase in fluorescence. The increase when ATP binds is found to occur in two phases in stopped-flow experiments. The slower phase has a rate close to that of the rapid 'burst' of $\mathrm{P}_{\mathrm{i}}$ production that occurs after the binding of ATP $\left[5 \mathrm{~s}^{-1}\right.$ (cold) to $200 \mathrm{~s}^{-1}$ (warm)] [5,6]; it has therefore been ascribed to a change at least in local structure accompanying the cleavage of ATP (step 3) [6]. However, Holmes [3] argued, on the basis of the atomic structures, that the fluorescence change occurs in an isomerization step that precedes and is required for cleavage; earlier work of Taylor and colleagues $[7,8]$ had similarly suggested that, at least with certain nucleotides, the increase in fluorescence occurs during an isomerization that precedes cleavage: that is, on the formation of an $\mathrm{M}^{* *}$.ATP state [9].

$$
\begin{aligned}
& \mathrm{M}+\mathrm{ATP} \underset{k_{-1}}{\stackrel{k_{1}}{\rightleftharpoons}} \text { M.ATP } \underset{k_{-2}}{\stackrel{k_{2}}{\rightleftharpoons}} \mathrm{M}^{*} \text {.ATP } \underset{k_{-3}}{\stackrel{k_{3}}{\rightleftharpoons}} \mathrm{M}^{* *} \text {.ADP.P } \|_{\mathrm{i}} \\
& \mathrm{M}+\mathrm{ADP} \underset{k_{-7}}{\stackrel{k_{7}}{\leftrightarrows}} \mathrm{M} . \mathrm{ADP} \frac{k_{6}}{\stackrel{\leftrightarrows}{k_{-6}}} \mathrm{M}^{*} \text {.ADP }+\mathrm{P}_{\mathrm{i}} \underset{k_{-5}}{\stackrel{k_{5}}{\leftrightarrows}} \mathrm{M}^{*} \text {.ADP.P }
\end{aligned}
$$

Scheme 1

Abbreviations used: $\mathrm{S} 1$, myosin subfragment 1 ; T-jump, temperature jump; $[\mathrm{NH}] \mathrm{ppA}$, adenosine $5^{\prime}$-[ $\beta, \gamma$-imido]triphosphate.

1 To whom correspondence should be addressed (e-mail wray@mpimf-heidelberg.mpg.de). 
Several steps in the myosin ATPase pathway are strongly dependent on temperature, especially the apparent forward rate of the cleavage step ( $k_{3}$ in Scheme 1) and the rate of ADP release (determined by $k_{6}$ ) [5]. Perturbing myosin-nucleotide complexes by rapid temperature jump (T-jump) is therefore an alternative approach to the study of the ATPase reaction and of the structural states occurring when various nucleotide and phosphate analogues are bound. Here we describe T-jump observations on the fluorescence of myosin S1 with several different ligands. The results show the existence of poised temperaturesensitive equilibria and provide new evidence regarding the isomerization of product and substrate states; they throw light on the occurrence under physiological conditions of the conformations seen by crystallography. Some results have already been reported in preliminary form $[10,11]$.

\section{EXPERIMENTAL}

\section{Proteins and nucleotides}

Rabbit and chicken S1 [12] was stored in concentrated solution with sucrose at $-80^{\circ} \mathrm{C}$. Dictyostelium motor fragment M765 [13] was a gift from D. Manstein. Nucleotides were purchased from Sigma.

\section{Solutions}

Proteins were dissolved in a standard buffer containing $70 \mathrm{mM}$ potassium propionate, $2-8 \mathrm{mM}$ magnesium acetate, $12 \mathrm{mM}$ Mops, $1 \mathrm{mM} \mathrm{NaN}_{3}$ and $5 \mathrm{mM}$ dithiothreitol, $\mathrm{pH}$ 7.0. The protein concentration was $0.5-5 \mu \mathrm{M}$. The change in buffer $\mathrm{pH}$ during the consumption of $1 \mathrm{mM}$ ATP was approx. 0.1 unit. To form the complexes with fluoride and aluminium or beryllium, the solutions contained $10 \mathrm{mM} \mathrm{KF}, 50 \mu \mathrm{M}$ ADP and $300 \mu \mathrm{M}$ $\mathrm{AlCl}_{3}$ or $\mathrm{BeCl}_{2}$ and were incubated at room temperature for at least $30 \mathrm{~min}$ before use. Nucleotides were in general used at saturating concentrations.

\section{Steady-state fluorescence}

Fluorescence was excited at 295 or $305 \mathrm{~nm}$; emission was monitored at $342 \mathrm{~nm}$ as the temperature of the cooling bath was changed continuously. With excitation at $305 \mathrm{~nm}$, the overall decline in fluorescence with increasing temperature was less steep, and the enhancement with ATP relative to ADP was larger, than with excitation at $295 \mathrm{~nm}$.

\section{Fluorescence T-jump}

T-jump kinetics were measured in a Garching Instruments (Garching, Germany) T-jump apparatus as described in [14]. The solution ( $3 \mathrm{ml}$ in a quartz cell with platinum electrodes) was heated by discharging $20 \mathrm{kV}$ from a $50 \mathrm{nF}$ capacitor with a heating decay time of approx. $10 \mu \mathrm{s}$. The amplitude of the Tjump was calibrated with a solution of Cresol Red in Tris $/ \mathrm{HCl}$ buffer and found to be $4{ }^{\circ} \mathrm{C}$. Fluorescence was excited with a $250 \mathrm{~W} \mathrm{Hg} / \mathrm{Xe}$ high-pressure arc lamp through a single monochromator (Schoeffel Instruments, Trappenkamp, Germany) at $295 \mathrm{~nm}$. Emission was monitored at right angles relative to the excitation beam through WG325/UG11 or WG305/UG12 (Schott, Mainz, Germany) filters. These filters cut off all light below 325 or $305 \mathrm{~nm}$ and above $400 \mathrm{~nm}$. After each T-jump the solution was left for 6-7 min to cool down to the starting temperature. The fluorescence signal suggested that the temperature remained effectively constant for $0.5-1 \mathrm{~s}$ after the $\mathrm{T}$ - jump; after that time, re-cooling and convectional flow inside the cell caused unpredictable changes in the signal that could not always be corrected for. Because under some conditions no relaxation slower than the heating process was seen at all, the transients did not seem to be subject to rotational orientation effects owing to the large electric fields during the capacitor discharge.

Data were acquired with a DL905 (Canberra Instruments, Munich, Germany) digital recorder for approx. $2 \mathrm{~ms}$ after the onset of capacitor discharge and at longer durations with a personal computer equipped with a DiSys analog-digital converter (DiSys, Düsseldorf, Germany). The data were smoothed by using a low-pass filter with a time constant at least 10 -fold faster than the relaxation time of the fastest relaxation process to be observed. Data were combined into a single data file with a logarithmic time scale and approximately 50 fluorescence readings for each order of magnitude on this scale.

In T-jump experiments with myosin, the heating of the sample was found to be accompanied by a fluorescence quench. This effect can be attributed to non-specific temperature dependence of the fluorescence quantum yield and was therefore not analysed in detail. Relaxation processes slower than the heating time of the instrument were in general interpreted as reflecting conformational transitions within the S1 molecule. No apparent deterioration occurred even in long series of jumps, and the transients shown in this paper are the result of summing approx. five to ten jumps. Transients were found to be independent of protein concentration in the range $1-5 \mu \mathrm{M}$ (thus suggesting that they were not due to temperature-induced aggregation). The temperatures indicated for $\mathrm{T}$-jumps refer to those before the jump.

Data were evaluated with the programme package Akkuprog [15] and the transients were characterized by fitting a sum of up to three exponentials. Allowance could also be made for cooling by allowing the baseline to rise linearly from the time of the jump; doing so had little effect on the derived parameters.

For some complexes studied, such as M.p[NH]ppA and M.ADP.BeF ${ }_{x}$, we found evidence for a simple two-state equilibrium. For this case, and assuming that the equilibrium constant $K$ varies with temperature in accordance with the van't Hoff equation, the amplitude of the relaxation after a T-jump is approximately proportional to $K /(1+K)^{2}$; it is therefore at a maximum when both states are equally populated (i.e. $K=1$ ). The equilibrium constant can be determined from the temperature dependence of this amplitude. If the amplitude changes only slightly over a large temperature range, this is an indication of a low reaction enthalpy.

\section{RESULTS}

\section{Steady-state fluorescence}

The dependence of the steady-state tryptophan fluorescence on temperature was studied in the range $4-30{ }^{\circ} \mathrm{C}$ for several myosinnucleotide complexes (Figure 1), extending an earlier study [16]. In general, an increase in temperature caused a decrease in steady-state fluorescence: only for ATP and CTP at low temperatures did the fluorescence increase.

The variation of fluorescence with temperature reflects two effects. One of these is the non-specific decline (see also the Experimental section) in fluorescence intensity with increasing temperature that is seen for many proteins and for tryptophan in solution: it reflects changes in the interaction of the tryptophan with neighbouring groups. A second effect is that the proportions of protein species with different fluorescences change with 


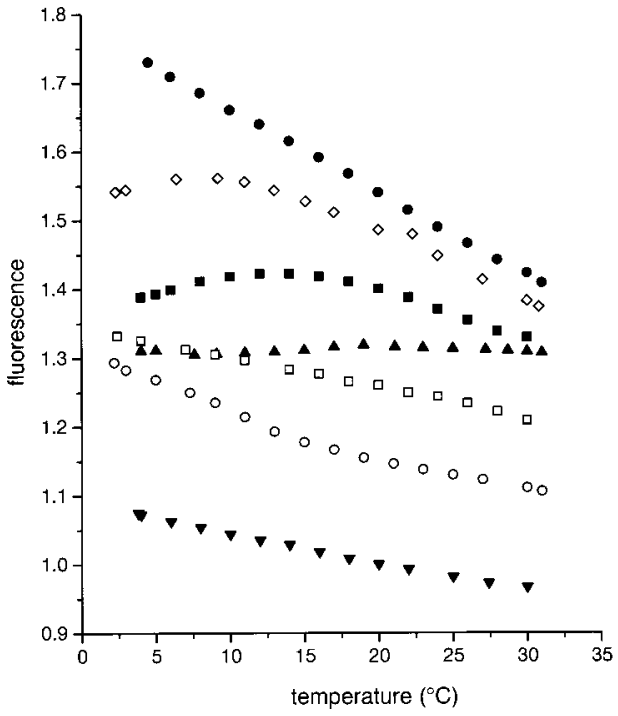

Figure 1 Temperature dependence of steady-state fluorescence of S1nucleotide complexes

Concentrations: $2 \mu \mathrm{M}$ rabbit $\mathrm{S} 1(\boldsymbol{\nabla})$, or $2 \mu \mathrm{M}$ rabbit S1 plus $100 \mu \mathrm{M}$ ADP $(\mathrm{O})$, initially $1 \mathrm{mM}$ ATP $(\boldsymbol{\square})$ or CTP $(\diamond), 200 \mu \mathrm{M} \mathrm{p}[\mathrm{NH}] \mathrm{ppA}(\mathbf{\Delta}), 500 \mu \mathrm{M}$ ADP plus $\left.1 \mathrm{mM} \mathrm{AICl}\right|_{3}$ plus $10 \mathrm{mM} \mathrm{KF}(\mathbf{O})$ or $100 \mu \mathrm{M}$ ADP plus $500 \mu \mathrm{M} \mathrm{BeCl}_{2}$ plus $10 \mathrm{mM} \mathrm{KF}(\square)$. Excitation was at $305 \mathrm{~nm}$; emission was measured at $342 \mathrm{~nm}$.

temperature. These two effects cannot be distinguished by inspection of the steady-state data. However, after a rapid Tjump the first (non-specific) effect is expected to cause a decrease in fluorescence with a time course not distinguishable from that of the T-jump itself; any change beyond this reveals the extent and rate of changes in the populations of different protein species.

\section{T-jump with no added nucleotide or with ADP}

A T-jump for our S1 preparations without added nucleotide showed only the expected non-specific decrease in intensity, occurring synchronously with the temperature rise; no sub- sequent process was resolvable under the conditions and at the temperatures used so far. The records resembled that illustrated for ADP. By contrast, T-jump observations [17] on S1 covalently modified with a fluorescent label suggested that two conformers interconverted on a time scale of $30 \mathrm{~ms}$. The behaviour of the complex M.ADP was studied at low $(50 \mu \mathrm{M})$ ADP, or more often after the complete hydrolysis of up to $1 \mathrm{mM}$ ATP to ADP and $\mathrm{P}_{\mathrm{i}}$ (Figure 2A). Under neither set of conditions was a transient clearly measurable at the temperatures $\left(4-20^{\circ} \mathrm{C}\right)$ studied.

\section{T-jump with ADP plus $\mathrm{BeF}_{x}$}

Figures 2(B)-2(D) show the result of adding Be and $F$ ions to a sample with ADP: a T-jump now caused a marked increase in fluorescence after the initial fall. Neither $\mathrm{BeCl}_{2}$ nor $\mathrm{KF}$ alone produced this transient, so the effect was a specific property of the M.ADP. BeF ${ }_{x}$ complex. Fitting this transient with a single exponential yielded a reciprocal time constant that varied somewhat with temperature (see Figure 4A). The amplitude varied approx. 1.5-fold over the range studied; it seemed to be maximal at approx. $14{ }^{\circ} \mathrm{C}$ (this value was $\mathrm{pH}$-sensitive, being higher at $\mathrm{pH} 6.5$ and lower at $\mathrm{pH}$ 7.7). There was sometimes evidence of a slower phase; however, this was too small in amplitude to be analysed further here.

\section{T-jump with $\mathrm{p}[\mathrm{NH}] \mathrm{ppA}$}

In the presence of $\mathrm{p}[\mathrm{NH}] \mathrm{ppA}$, which is hydrolysed only extremely slowly by myosin, a clear transient was again seen (Figure 3 ). The principal transient did not change as $\mathrm{p}[\mathrm{NH}] \mathrm{ppA}$ concentration was varied from 6 to $250 \mu \mathrm{M}$; it therefore did not seem to reflect a temperature dependence in the degree of saturation of the protein with the nucleotide. The reciprocal time constant was in the range $700-1200 \mathrm{~s}^{-1}$ (Figure 4A), and the amplitude varied up to 2-fold over the temperature range studied; it was maximal at approx. $13{ }^{\circ} \mathrm{C}$. As with $\mathrm{ADP} . \mathrm{BeF}_{x}$, there was sometimes evidence of a much slower phase of much lower amplitude.

\section{T-jump in the presence of ATP}

Addition of ATP to the S1 sample produced a different response to a T-jump. In a typical experiment, $1 \mathrm{mM}$ ATP was added and
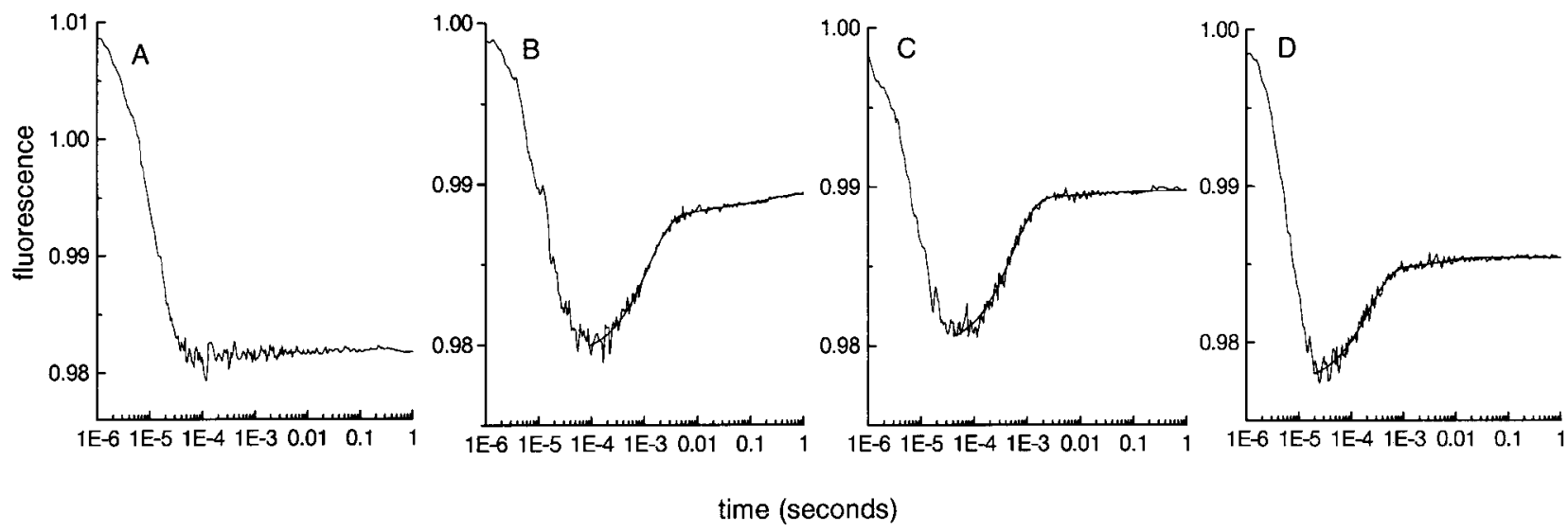

Figure 2 T-jump responses in ADP and in ADP plus $B F_{x}$

(A) Response to a $4{ }^{\circ} \mathrm{C} \mathrm{T-jump} \mathrm{for} \mathrm{chicken} \mathrm{S} 1+50 \mu \mathrm{M}$ ADP at a pre-jump temperature of $4{ }^{\circ} \mathrm{C}$. Here and in subsequent Figures, fluorescence is plotted relative to the intensity immediately before the T-jump. (B-D) T-jump transients for rabbit S1 after the addition of $10 \mathrm{mM} \mathrm{KF}$ and $330 \mu \mathrm{M} \mathrm{BeCl}{ }_{2}$ to $4 \mu \mathrm{M} \mathrm{S} 1$ plus $50 \mu \mathrm{M} \mathrm{ADP}$, for pre-jump temperatures of $3{ }^{\circ} \mathrm{C}(\mathbf{B}), 15^{\circ} \mathrm{C}(\mathbf{C})$ and $24.5^{\circ} \mathrm{C}$ (D). $1 \mathrm{E}-6=1 \times 10^{-6}$ etc. 


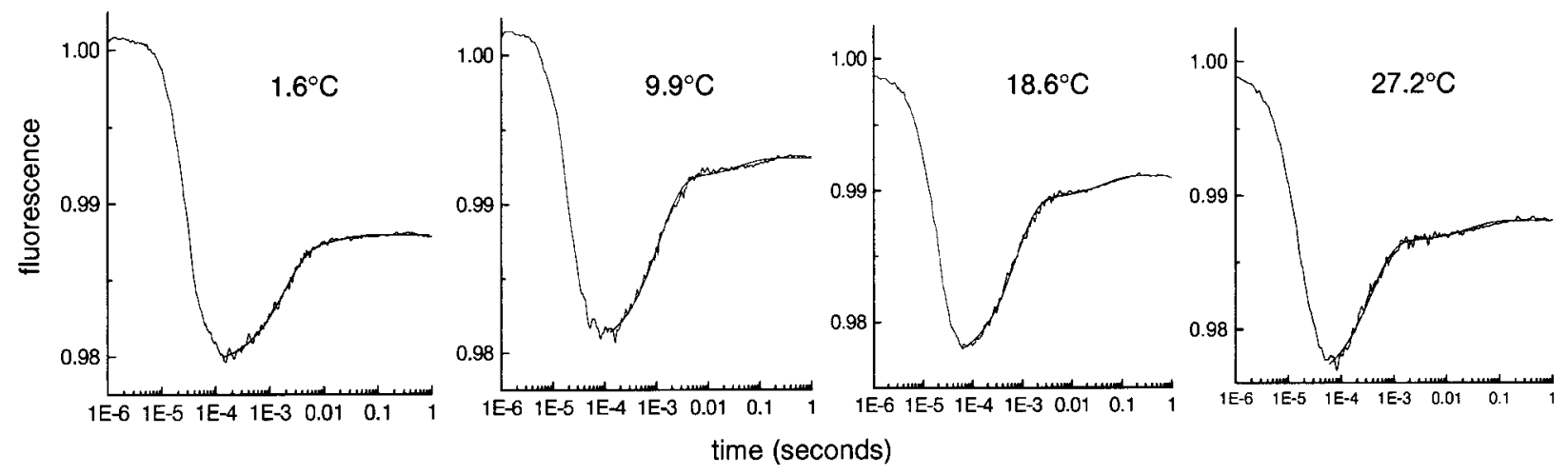

Figure 3 T-jump responses in $\mathrm{p}[\mathrm{NH}] \mathrm{ppA}$

Transients are shown for rabbit S1 $(4 \mu \mathrm{M})$ plus $160 \mu \mathrm{M}$ p[NH]ppA at the four pre-jump temperatures indicated. $1 \mathrm{E}-6=1 \times 10^{-6}$ etc.

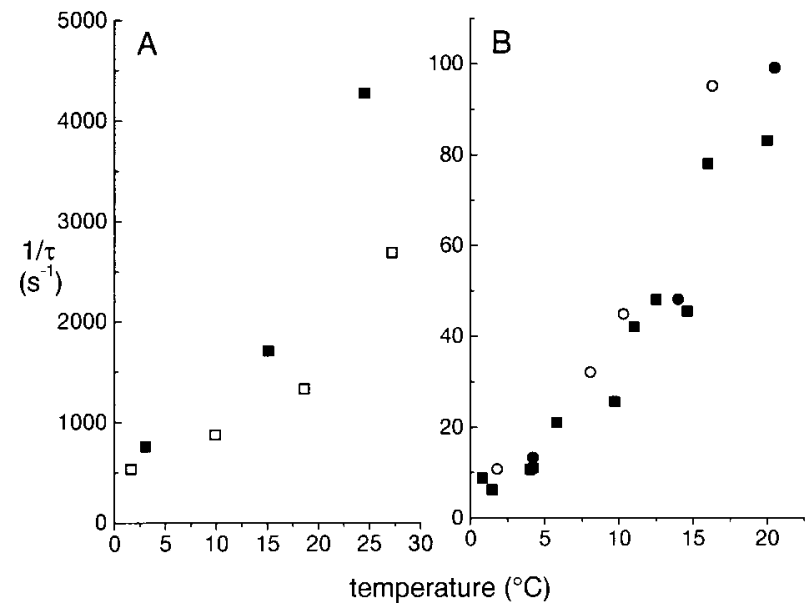

Figure 4 Reciprocal time constants for T-jump transients

The abscissa indicates temperatures measured before each $4{ }^{\circ} \mathrm{C}$ jump. (A) Reciprocal time

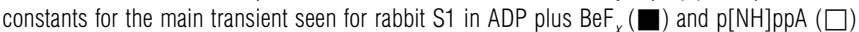
(experiments in Figures 2 and 3). (B) Reciprocal time constant determined for the main phase of the responses to T-jump during ATP hydrolysis for rabbit S1 ( $\mathbf{\square})$ and for chicken isoforms $\mathrm{A1}(-)$ and $\mathrm{A2}(\mathrm{O})$ (summary of results from a series of measurements with several different S1 preparations).

measurements were made at intervals as it was consumed by hydrolysis (over 2-3 h). The transients seen in the presence of ATP could not be fitted by single exponentials (Figure 5), but instead required at least three, extending to much slower regions than for $\mathrm{p}[\mathrm{NH}] \mathrm{ppA}$ and $\mathrm{ADP}+\mathrm{BeF}_{x}$. Over most of the long period during which ATP was consumed, no change was seen: only in the few minutes before ATP was exhausted (as confirmed by HPLC) did the relaxations disappear as expected for S1 with ADP. The T-jump response therefore did not seem to be affected by the concentration of unbound ATP.

The largest and best-characterized phase (the main phase) of the transient showed a reciprocal time constant that varied greatly with starting temperature, from 10 to $100 \mathrm{~s}^{-1}$ (Figure 4B). Very similar rates were found for chicken and rabbit $\mathrm{S} 1$; the isoforms $\mathrm{A} 1$ and $\mathrm{A} 2$ did not seem to differ. Within the region around $\mathrm{pH} 7$, the solution $\mathrm{pH}$ had a small effect on timeconstant, whereas varying the free $\mathrm{Mg}^{2+}$ concentration $(2-7 \mathrm{mM}$ ) had no effect. The amplitude of this phase declined steadily with rising temperature, so that above $20^{\circ} \mathrm{C}$ the phase was hard to characterize.

A fast phase was seen at all temperatures. Its apparent amplitude was approx. $20 \%$ of that of the main phase (see legend to Figure 5); this phase was more difficult to characterize quantitatively because of the relative noisiness of the data at short durations. Its amplitude might be underestimated, particularly at higher temperatures. Its existence was nevertheless clear from comparison with results in ADP or without nucleotide. In respect of its time constant, this transient was similar to, or somewhat faster than, the much larger principal transients seen with $\mathrm{p}[\mathrm{NH}] \mathrm{ppA}$ and ADP. $\mathrm{BeF}_{x}$.

A prominent slow phase was observed, with a reciprocal time constant of approx. $1 \mathrm{~s}^{-1}$ in the warm. The change in the signal in this time-region could not be explained merely by re-cooling and it required a third exponential; however, the start of the cooling process meant that this relaxation could not be reliably followed to completion. The time constant increased markedly at lower temperatures and therefore became undeterminable under our conditions. The amplitude of the slow phase was comparable with that of the main phase and increased at lower temperatures.

\section{Other ligands}

Several other nucleotides and analogues were investigated. The complex formed with aluminium and fluoride ions (M.ADP. $\mathrm{AlF}_{4}{ }^{-}$) had a higher steady-state fluorescence than the complex with ADP and $\mathrm{BeF}_{x}$ but showed no transient at temperatures studied so far (results not shown).

Figure 6 shows transients in the presence of $\mathrm{XDP}$ and $\mathrm{AlF}_{4}^{-}$. These were fitted well by single exponentials; they were an order of magnitude slower than for ADP. BeF and were seen only at lower temperatures. This behaviour illustrates the point that the existence of two forms of a complex can be overlooked if the equilibrium is displaced too far towards one form under the conditions of observation.

Figure 7(A) shows a transient recorded in CTP at $0{ }^{\circ} \mathrm{C}$. This showed the three phases seen for ATP. With CTP at temperatures above $10{ }^{\circ} \mathrm{C}$, the transient was no longer seen.

\section{Dictyostelium myosin motor domain}

We made a smaller number of measurements with expressed Dictyostelium myosin motor domain (residues 1-765) lacking the light-chain-binding region. The behaviour of this myosin was of interest in interpreting the fluorescence signals because of the 


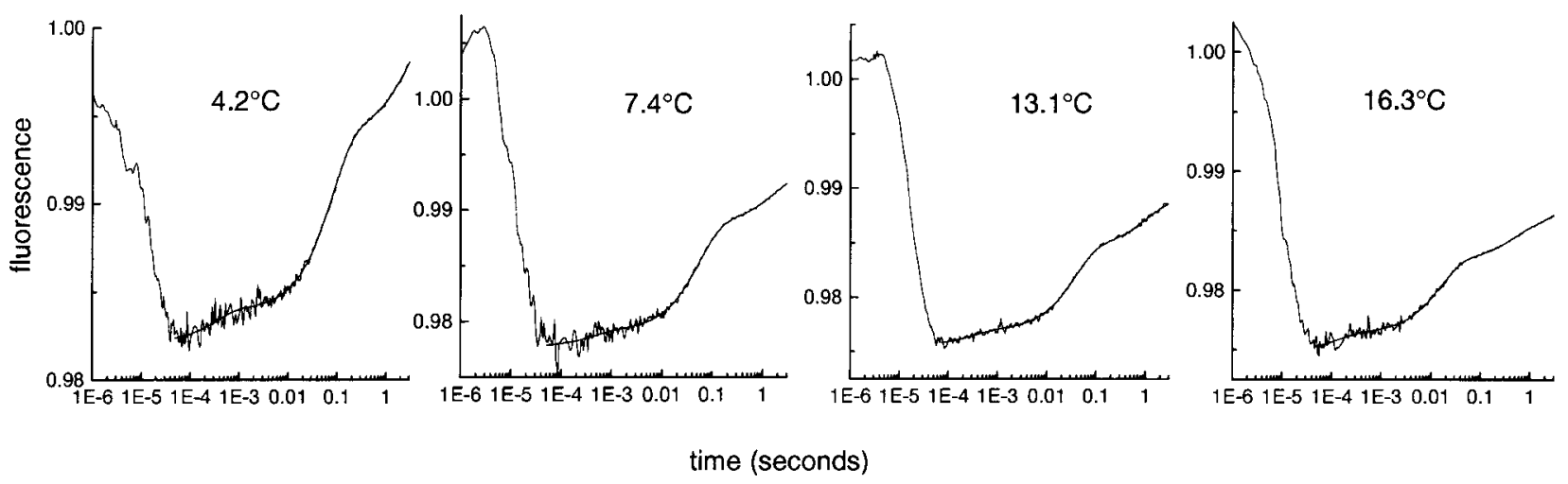

\section{Figure 5 T-jump transients in ATP}

Transients are shown for chicken $\mathrm{S} 1(4 \mu \mathrm{M})$ during the steady-state hydrolysis of initially $1 \mathrm{mM}$ ATP, for the four different pre-jump temperatures indicated. The three exponentials (fast, main and slow) fitted to these transients had respective reciprocal time constants as follows (in s-1): 2500,13 and 0.67 at $4{ }^{\circ} \mathrm{C}, 2500,18.2$ and 0.66 at $7.4^{\circ} \mathrm{C}, 3300,27$ and 1.12 at $13^{\circ} \mathrm{C}$, and $6700,62.5$ and 2.3 at $16.3^{\circ} \mathrm{C}$. Amplitudes of the fast phase relative to the main phase were $0.18,0.17,0.18$ and 0.25 at the four temperatures respectively. $1 \mathrm{E}-6=1 \times 10^{-6}$ etc.
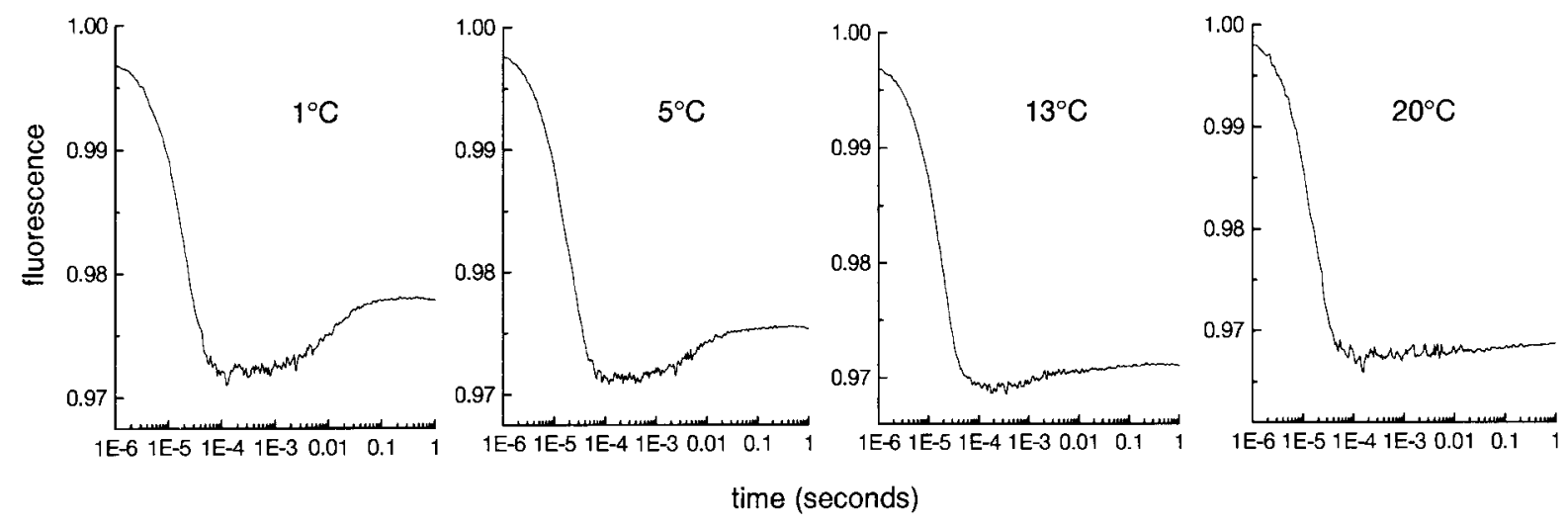

\section{Figure 6 T-jump responses in XDP and AlF $_{4}^{-}$}

Transients for rabbit $\mathrm{S} 1(2 \mu \mathrm{M})$ in $100 \mu \mathrm{M}$ XDP plus $330 \mu \mathrm{M} \mathrm{AICl}$ plus $10 \mathrm{mM} \mathrm{KF}$ at the four different pre-jump temperatures shown. $1 \mathrm{E}-6=1 \times 10^{-6}$ etc.
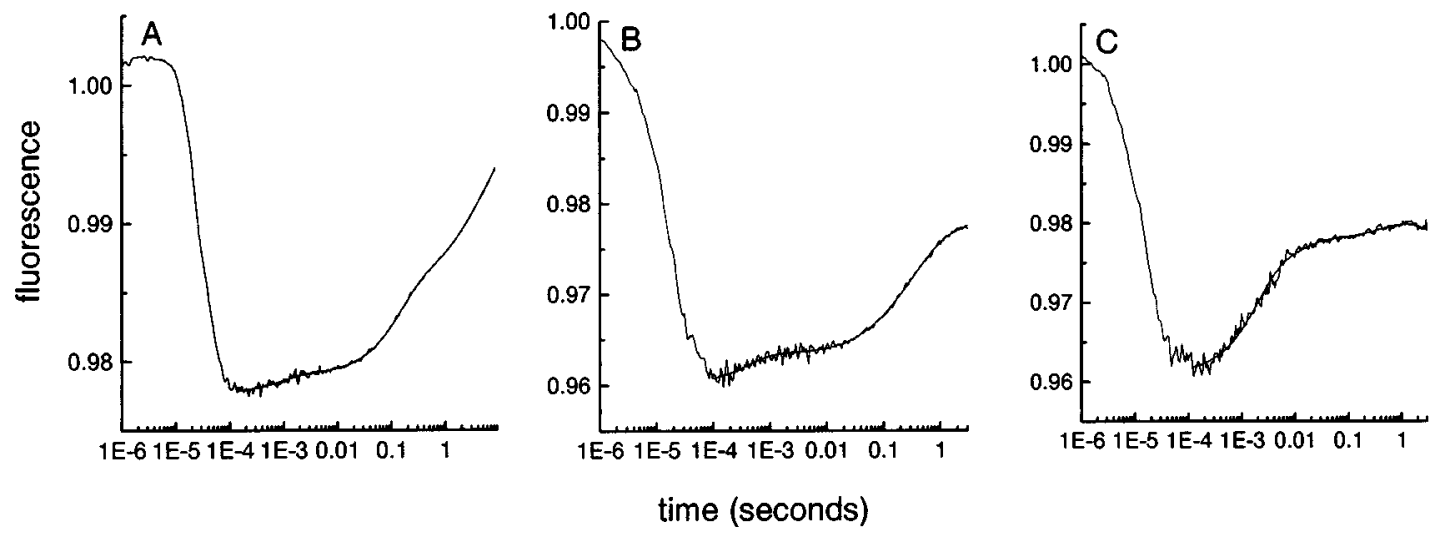

\section{Figure 7 Further T-jump transients}

(A) T-jump response for chicken S1 during the hydrolysis of $150 \mu \mathrm{M}$ CTP; pre-jump temperature $0{ }^{\circ} \mathrm{C}$. Analysis of this transient yielded phases with reciprocal time constants of $1031,6.97$ and $0.481 \mathrm{~s}^{-1}$. (B, C) T-jumps for Dictyostelium motor domain $(5 \mu \mathrm{M})$, at pre-jump temperatures of $6.5^{\circ} \mathrm{C}(\mathbf{B})$ and $5.4^{\circ} \mathrm{C}(\mathbf{C})$ during the hydrolysis of initially $500 \mu \mathrm{M}$ ATP $(\mathbf{B})$ and subsequently in $500 \mu \mathrm{M}$ ADP plus $500 \mu \mathrm{M} \mathrm{P}$, plus $330 \mu \mathrm{M} \mathrm{BeCl}_{2}$ plus $10 \mathrm{mM} \mathrm{KF}(\mathbf{C})$. The principal transients had reciprocal time constants of $12.6 \mathrm{~s}^{-1}$ in $(\mathbf{B})$ and $515 \mathrm{~s}^{-1}$ in $(\mathbf{C}) .1 \mathrm{E}-6=1 \times 10^{-6}$ etc. 
different locations of its tryptophan residues. T-jumps were recorded without added nucleotide, during the steady-state turnover of ATP, and in the presence of ADP or ADP.BeF ${ }_{x}$; the results with ATP and with $\mathrm{ADP} . \mathrm{BeF}_{x}$ (Figures $7 \mathrm{~B}$ and $7 \mathrm{C}$ ) closely resembled those for skeletal $\mathrm{S} 1$.

\section{Relation of T-jump transients to steady-state data}

The amplitudes of changes in fluorescence after a T-jump corresponded to those expected for $4{ }^{\circ} \mathrm{C}$ jumps on the basis of the steady-state fluorescence levels in Figure 1. Comparison of the steady-state and T-jump data shows that transients after a Tjump were largest for those complexes, and at those temperatures, for which the slope of the steady-state curve departed most strongly from the downward slope seen with ADP and without nucleotide. This behaviour suggests that the latter downward slope reflected the temperature dependence of the fluorescence of individual states and that departures from this slope resulted when a temperature change also caused a shift in the proportions of states with different fluorescences. In all cases described here, this departure was in the direction of less downward or even an upward (ATP, CTP) slope. Correspondingly, in the T-jump data, when transients were seen after the initial decrease, they were always transient increases in fluorescence.

\section{DISCUSSION}

In this study we examined the response of several myosinnucleotide complexes to rapid T-jumps. The transient changes in the tryptophan fluorescence of myosin $\mathrm{S} 1$ after a $\mathrm{T}$-jump reveal that under certain conditions the protein is present as an equilibrium mixture of more than one species, as judged from the perturbability of the signal. These findings, together with the variation in steady-state fluorescence with temperature, provide new information on the existence and interconversion kinetics of different structural states of myosin in these complexes.

The simplest transients were seen with $\mathrm{p}[\mathrm{NH}] \mathrm{ppA}$ and ADP. BeF ${ }_{x}$ bound; they are explained well in both cases by a poised equilibrium between two states with different fluorescences. The equilibrium constant was 1 in the neighbourhood of $15^{\circ} \mathrm{C}$ for both ligands; on the simplest model (see the Experimental section), the results with $\mathrm{ADP} . \mathrm{BeF}_{x}$ suggest a change in $K$ from 0.2 to 0.5 , and for $\mathrm{p}[\mathrm{NH}] \mathrm{ppA}$ from 0.2 to 2 , over the temperature range studied, which is also consistent with the steady-state fluorescence for these ligands (Figure 1). Interconversion occurred rapidly (reciprocal time constant approx. $10^{4} \mathrm{~s}^{-1}$ at room temperature, with only a slight dependence on temperature). This suggests, for both ligands, a very small enthalpy and free energy difference and a low activation enthalpy, so that both species were substantially represented throughout the temperature range studied. It is likely, but not proved, that the two states are the same two conformations in both of these analogues. We note in passing that, in any kinetic system, evidence for a two-state equilibrium is in general also interpretable in terms of a continuum of states. We have no independent evidence for or against this formal possibility for myosin-nucleotide complexes.

\section{Transients during steady-state hydrolysis of ATP}

In the presence of ATP the transients are more complex. Three phases were reproducibly observed; only the middle one was easily characterizable, because the faster one was not sufficiently resolved from the T-jump and the slower one extended into the time range (beyond $1 \mathrm{~s}$ ) where re-cooling was significant. The

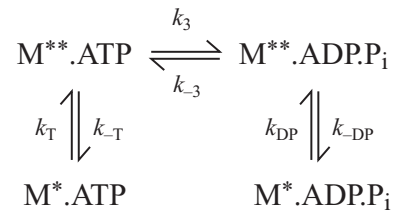

Scheme 2

existence of these phases indicates an equilibration between more than two states, as is indeed to be expected, because in the Bagshaw-Trentham scheme at least three different chemical species are present in substantial amounts during the steady-state hydrolysis of ATP.

According to Scheme 1, under conditions of excess ATP, two very slow processes are the release of $P_{i}$ and of ADP. The rate constant that limits ADP release $\left(k_{6}\right)$ declines with decreasing temperature much more than that for $\mathrm{P}_{\mathrm{i}}$ release $\left(k_{4}\right)$, so the steady-state concentration of M.ADP increases at low temperatures [4,5]. A T-jump is therefore followed by a decrease in [M.ADP], at a rate that depends on $\left(k_{4}+k_{6}\right)$. Because M.ADP has a lower fluorescence than the mixture of triphosphate states, this process can well explain the slow phase of fluorescence increase that we observe in the presence of ATP.

The Bagshaw-Trentham scheme, in the form of Scheme 1, implies a direct coupling between the cleavage step and the change to a state of higher fluorescence. The cleavage equilibrium $\left(K_{3}\right)$ seemed to be temperature-sensitive, the products complex being favoured at higher temperature [9]. Scheme 1 predicts that a T-jump should cause a transient increase in fluorescence, as $\left[\mathrm{M}^{*}\right.$.ATP $]$ decreases and $\left[\mathrm{M}^{* *}\right.$.ADP.P $\left.\mathrm{P}_{\mathrm{i}}\right]$ increases, with a rate constant reflecting $\left(k_{3}+k_{-3}\right)$. This would indeed explain the observed properties of the main phase.

However, it would not in itself explain the faster phase as well, so we consider the alternative represented by Scheme 2. Here, both M.ATP and M.ADP.P. i $_{i}$ exist as two conformations with different fluorescences, both of which are significantly populated in the steady state (that is, with equilibrium constants $K_{\mathrm{T}}$ and $K_{\mathrm{DP}}$ close to 1). We use asterisks to distinguish these states because we are still concerned with interpreting the fluorescence changes that are indicated in this way in Scheme 1. It is also important to consider the possibility $[3,18]$ that only one state is capable of hydrolysing ATP (i.e. the interconversion of $\mathrm{M}^{*}$.ATP and $M^{*}$.ADP.P can $_{\mathrm{i}}$ be neglected).

Such a scheme can in principle be thermodynamically uncoupled, meaning that $K_{\mathrm{T}}=K_{\mathrm{DP}}$. However, the general case is that of partial coupling, the two conformations existing both for product and for substrate complexes but in different proportions $\left(K_{\mathrm{T}} \neq K_{\mathrm{DP}}\right)$.

This type of scheme allows for the appearance of further phases in T-jump, depending on which step or steps are sensitive to temperature. Many combinations are possible here. One simple example of the production of a fast phase is that $K_{\mathrm{T}}$ and $K_{\mathrm{DP}}$ are rapid equilibria but $K_{\mathrm{DP}}$ is larger than $K_{\mathrm{T}}$ (i.e. M.ADP.P is more strongly in state $\mathbf{M}^{* *}$ than M.ATP is) and $\mathbf{M}^{* *}$ is the only state in which cleavage is possible. A T-jump will then produce a fast initial increase in $\mathrm{M}^{* *}$.ATP at the expense of $\mathrm{M}^{*}$.ATP; a further re-equilibration between $\mathrm{M}^{* *}$.ATP and $\mathrm{M}^{*}$.ATP then follows to replace the $\mathrm{M}^{* *}$.ATP as it decays to $\mathrm{M}^{* *}$.ADP.P $\mathrm{P}_{\mathrm{i}}$, this second phase being limited by the much lower rate of the cleavage step.

This explanation is directly suggested by our findings with the two analogues studied ( $[\mathrm{NH}] \mathrm{ppA}$ and $\mathrm{ADP} . \mathrm{BeF}_{x}$ ), because 


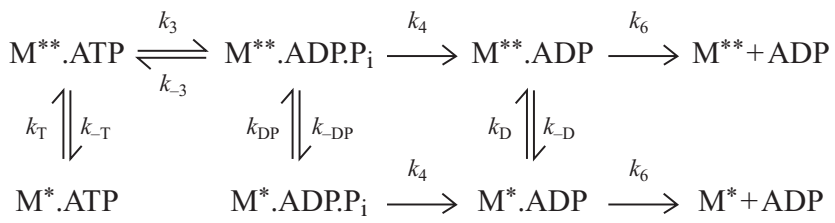

Scheme 3

these complexes yielded transients similar in time constant to the fast phase in ATP. However, it is not the only explanation; thus some sensitivity to temperature could occur in $K_{3}$ as well. Other combinations, such as a sensitivity of $K_{\mathrm{DP}}$ to temperature, would produce a main phase of reverse sign.

\section{Modelling of ATP transients}

To make these considerations explicit, we computed T-jump transients expected on the basis of Scheme 2 together with product release steps as shown in Scheme 3. The purpose of the simulations was to understand the implications of Scheme 2 rather than to produce a unique fit of the calculated to the measured transients. T-jumps simulated with Scheme 3 were analysed and found, like the measured ones, to be fittable as the sum of three exponentials. Each of the reciprocal time constants derived for a simulated transient in principle depends on all of the rate constants in the scheme and does not correspond to any one of them directly. Rate constants for the scheme were sought such that the simulated and observed transients exhibited similar time constants. Figure 8 shows simulations of $\mathrm{T}$-jumps for skeletal S1 in ATP from 3 to $7^{\circ} \mathrm{C}$ and from 16 to $20^{\circ} \mathrm{C}$, on the basis of the argument in the previous section and for the case in which $K_{\mathrm{T}}$ and the forward and backward rates of the cleavage step are sensitive to temperature but $K_{3}$ is not. The kinetic parameters are indicated in the legend to Figure 8 and were also such as to be consistent with parameters of the myosin ATPase (including product release steps) for skeletal S1 derived from other methods [4,5], under solution conditions as close as possible to our own. Our T-jump data in ATP are clearly adequately explained on the basis of the Scheme 3. Note that some constants are not strongly constrained by the modelling. The equilibrium between single-starred and double-starred complexes for M.ADP.P ${ }_{i}$ is included by analogy with the other complexes; it is assumed to be fast and our data indicate only that $K_{\mathrm{DP}}$ is larger than $K_{\mathrm{T}} ; K_{\mathrm{d}}$ was assumed to be much less than 1 .

It is important to test whether the kinetic scheme implied by our T-jump data can also account for the $\mathrm{P}_{\mathrm{i}}$ burst and the fluorescence changes that occur on binding of ATP $[4,6]$. In simulations of these processes (Figures 8C and 8D) the fluorescence change is seen to occur in two phases. The first phase would not be visible, lying within the $1 \mathrm{~ms}$ stopped-flow dead time together with the presumed change associated with the irreversible step 2. The slower phase is similarly due to a fluorescence change on isomerization; the strong temperature dependence of the rate of this phase is partly due to that of $K_{\mathrm{T}}$ but its rate in simulations varied with the assumed values of $\left(k_{3}+k_{-3}\right)$. In the simulations the slow fluorescence and $\mathrm{P}_{\mathrm{i}}$ burst curves have very similar rates (see legend to Figures $8 \mathrm{C}$ and $8 \mathrm{D}$ ) and both agree with published data, particularly when the effect of ionic conditions is considered $[5,6]$. The slow phase in our modelling arises because of the fast and reversible isomerization preceding cleavage, for which our perturbation approach provides evidence. This explanation differs qualitatively from two previous ones,
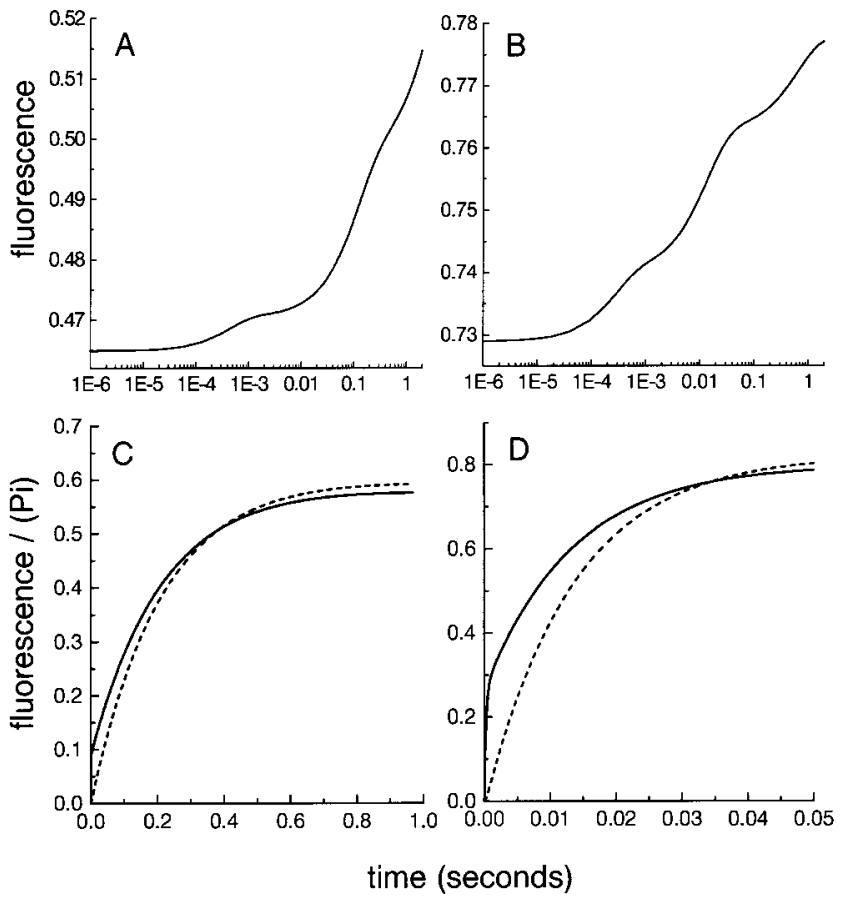

Figure 8 Simulations based on Scheme 3

(A, B) Model calculations showing the response of the tryptophan fluorescence to a T-jump on the basis of Scheme 3, plotted on a logarithmic time scale for comparison with the experimental transients. Simulations are for jumps from 3 to $7^{\circ} \mathrm{C}(\mathbf{A})$ and from 16 to $20^{\circ} \mathrm{C}(\mathbf{B})$. The ordinate shows percentage of the protein in the state $(* *)$ with higher fluorescence. Rate constants $\left(\mathrm{s}^{-1}\right)$ were as follows at $3^{\circ} \mathrm{C}$ (in parentheses; values for $20^{\circ} \mathrm{C}$ ): $k_{3} / k_{-3}: 32.1 / 2.14(224.6 / 15)$; $k_{T} / k_{-T}: 200 / 2000(1000 / 2500) ; k_{D P} / k_{-D P}: 2000 / 200(2000 / 200) ; k_{4}: 0.053(0.06) ; k_{D} / k_{-D}$ : $0.1 / 10(0.1 / 10) ; k_{6}: 0.125(1.5)$. Parameters at the intermediate temperatures required in simulating the jumps were obtained by interpolation between the values for 3 and $20^{\circ} \mathrm{C}$ in accordance with van't Hoff and Arrhenius equations. The fast phase has an amplitude relative to the main phase of 0.21 in $(\mathbf{A})$ and 0.49 in $(\mathbf{B})$. (C, D) Simulations (linear time scale) of the fluorescence enhancement after binding ATP (full line) and the progress curve of the $P_{i}$ burst (broken line), based on the states in Scheme 3. Curves are shown for $3^{\circ} \mathrm{C}(\mathbf{C})$ and $20^{\circ} \mathrm{C}(\mathbf{D})$ (note different time scales). The fluorescence curve is derived as the change in the fraction of the protein in the double-starred state $(* *)$, starting from exclusively $M^{*}$.ATP as the initially formed state; its magnitude is arbitrary because the difference between $M^{*}$ and $M^{* *}$ and the increase on the formation of $\mathrm{M}^{*}$.ATP are not specified. At both temperatures, a very rapid initial fluorescence rise is followed by a slower one whose rate depends on the cleavage step. Fitting of exponentials to these simulated time courses yielded: $P_{i}$ rate $4.86 \mathrm{~s}^{-1}$ (cold) and $73.8 \mathrm{~s}^{-1}$ (warm) and slow fluorescence rate of $4.9 \mathrm{~s}^{-1}$ (cold) and $74.5 \mathrm{~s}^{-1}$ (warm). Simulations (A-D) were made with the program KSIM written by N. Millar (http://wuarchive.wustl.edu/ packages/kinsim/uploads/). $1 \mathrm{E}-6=1 \times 10^{-6}$ etc.

namely that the slow fluorescence reflects cleavage directly and therefore has the same rate [6], or that isomerization occurs at the rate observed for slow fluorescence change, whereas the cleavage equilibrium is much faster and thus does not appreciably delay the $\mathrm{P}_{\mathrm{i}}$ burst curve [1].

In the presence of CTP, transients were seen only at temperatures up to $10^{\circ} \mathrm{C}$ and showed the same three phases as for ATP. The apparent cleavage equilibrium for CTP is shifted towards the products complex compared with ATP [19]. Both observations are explained in terms of our present model if $K_{\mathrm{T}}$ is larger for CTP than for ATP at the same temperature.

\section{Relationship to known S1 conformations}

The above arguments show that our findings, taken together, can be explained well in terms of two myosin-ligand conformations 
$\mathrm{M}^{*}$ and $\mathrm{M}^{* *}$, such that the bound ligand affects the proportions of the two conformations but not the fluorescence of each. The equilibrium between the two conformations is affected by temperature, thus making our present perturbation approach possible. Our observations relate exclusively to the tryptophan fluorescence of myosin. Changes in this signal do not necessarily indicate larger conformational differences, because one tryptophan residue $\left(\operatorname{Trp}^{131}\right)$ is in the immediate vicinity of the nucleotide-binding site in skeletal S1. In Dictyostelium S1, the corresponding tryptophan residue is missing; changes in tryptophan fluorescence in Dictyostelium might therefore indicate a structural change that is not confined to the active site $[1,20]$. Our Dictyostelium T-jump data closely resemble those from skeletal $\mathrm{S} 1$, thereby suggesting that the $\mathrm{T}$-jump transients in both systems characterize large-scale conformational transitions in myosin. The different fluorescence states could therefore correspond to the two forms in which M.ADP. BeF ${ }_{x}$ has been found by crystallography ([21], and J. Kull, personal communication), which were termed 'down' and 'up' by Holmes [3], corresponding to 'open' and 'closed' [1]; the transition between them might be sensed particularly by $\operatorname{Trp}^{501}$. A recent mutational study of Dictyostelium myosin motor domain [22], separating the contributions of individual tryptophans, provides further evidence that the fluorescence of $\operatorname{Trp}^{501}$ signals the open-closed transition. Our present results provide the first direct evidence on the kinetics of interconversion of these states under solution conditions.

M.p[NH]ppA has so far been found only in the 'down' form crystallographically [23]; our results therefore imply that the 'up' form might be found with this ligand at higher temperature. In agreement with this, the fluorescence of Trp ${ }^{501}$ from Dictyostelium was strongly dependent on temperature when $\mathrm{p}[\mathrm{NH}] \mathrm{ppA}$ was bound [22]. The identification of $\mathbf{M}^{* *}$ with 'up' and $\mathbf{M}^{*}$ with 'down' remains a plausible working hypothesis, which we employ for the remainder of this discussion. However, the possibility of a contribution from further states, such as the 'detached' state hitherto seen only in scallop S1 [2], cannot be excluded. The physical basis of the temperature dependence is still not clear from the atomic structures.

Our results provide evidence for the existence of a corresponding thermodynamic equilibrium between two forms of S1 with ATP bound, independently of the hydrolysis step. Crystallographic evidence has been presented [18] for the existence of M.ATP in the 'down' conformation, perhaps constrained by the crystal packing. No direct crystallographic evidence has yet been found for a conformation of either M.ATP or M.ADP.P ${ }_{i}$ corresponding to 'up', as implied by our results (but see also $[24,25])$. Our results show that the tendency to form the highfluorescence state is greater in M.ADP.P ${ }_{i}$ than in ATP (see also [22]). The species $M^{*}$.ADP. $P_{i}$ was included in Scheme 1 [4] to accommodate other evidence, namely for a state that binds and releases $\mathrm{P}_{\mathrm{i}}$ more rapidly than the predominant intermediate $M^{* *}$.ADP. $P_{i}$; product release from M.ADP. $P_{i}$ in the absence of actin might indeed occur exclusively from the small fraction that is in the 'open' conformation.

In contrast with these ligands, the complex M.ADP.AlF ${ }_{4}^{-}$gave T-jump transients suggesting that only a single conformation was substantially present under the conditions studied so far. Because of the high fluorescence, and because the 'up' form was found by crystallography even at low temperature [21], this complex is presumably 'up'. Our result with $\mathrm{XDP} \mathrm{AlF}_{4}{ }^{-}$suggests that complexes with $\mathrm{AlF}_{4}^{-}$do not invariably induce this conformation.

With ADP, where with skeletal S1 we found no clear transient, the low fluorescence suggests that the 'down' state predominates. However, in other myosins we have found evidence for transitions with ADP bound; further studies might well reveal conditions in which such transitions also occur for skeletal S1.

\section{Implications for coupling in myosin}

Structural interconversion of myosin-ligand complexes is relevant to events surrounding the chemical step of ATP cleavage. This step is easily reversible, so that the free energy difference between ATP and ADP plus $\mathrm{P}_{\mathrm{i}}$ in solution must be balanced by their greatly different binding energies to the protein; cleavage therefore involves the transduction of free energy into a (chemically or mechanically) different form. The substrate and product complexes will have different structures, which are crucial partners in this transduction process. Components of the structural difference might, for example, be the distance between $\beta$ - and $\gamma$-phosphates or changed protein-ligand interactions. Lymn and Taylor [26] proposed that cleavage was coupled not only to such local changes but also to a larger conformational transition, as implied by the fluorescence signal. The different structures observed in the present study need to be considered in this context.

Much work in this area, like our own, has employed analogues, with the aim of dissecting different aspects of the behaviour of the physiological substrate ATP, although no analogue can be identical with ATP in any respect. The two states exist, for $\mathrm{p}[\mathrm{NH}] \mathrm{ppA}$ and $\mathrm{ADP} . \mathrm{BeF}_{x}$, in a poised equilibrium over a large temperature range. This suggests that $\mathrm{p}[\mathrm{NH}] \mathrm{ppA}$ and $\mathrm{ADP} . \mathrm{BeF}_{x}$ produce the 'up' conformation at sufficiently high temperature. The protein structure is not known for $\mathrm{p}[\mathrm{NH}] \mathrm{ppA}$ in the 'up' state, but presumably the transition is not coupled to hydrolysis of the PN-P bond. With ADP. BeF ${ }_{x}$ both forms have been observed; in the 'up' state determined for a truncated motor domain with ADP. $\mathrm{BeF}_{x}$ bound the geometry surrounding the $\gamma$ phosphate position does not seem to differ from that seen in the 'down' form [21] (J. Kull, personal communication). We interpret our results with ATP to mean that M.ATP similarly exists in at least two states. Thus the isomerization between the two S1 structures is remarkably constant for a range of ligands of strongly varying binding affinities. It will be important to understand this feature in terms of the atomic structures of myosin-ligand complexes.

Whether 'up'or 'down' is favoured does not seem to be related either to a change in the separation of the $\beta$ - and $\gamma$ phosphates or to the affinity of the bound ligands. Neither does the 'up' conformation seem to be specifically associated with the binding of products rather than substrate, i.e. with storage of the energy of ATP hydrolysis. This concept would be plausible if the coupling were rigid, but it becomes untenable as the degree of coupling in Scheme 2 decreases. The two conformations are also not correlated with weak and strong binding to actin [1]; both bind weakly to actin, and the mechanism by which the ligand affects the strength of attachment to actin is apparently independent of the up-down equilibrium.

The transition might nevertheless be related to the power stroke in the sense that it reverses one component of it after detachment. Indeed, Lymn and Taylor viewed the hydrolysis step as re-establishing a cross-bridge conformation able to perform a power stroke. Little evidence for changes associated with cleavage, or indeed for alternative cross-bridge conformations at all in the absence of actin, was available before crystallography revealed the atomic structures. However, steadystate and T-jump measurements with X-ray diffraction from myosin filaments in intact muscle [27-29] had suggested a temperature-dependent equilibrium between two cross-bridge structures, seemingly related to cleavage as envisaged by Lymn 
and Taylor; this equilibrium is probably related to the isomerization discussed here. Our present results clarify this aspect of the Lymn-Taylor scheme for single S1 molecules. The coupling is not rigid. Thus cleavage does promote the tendency of the head to be in the 'up' conformation; however, even ATP, the most tightly binding ligand, induces two different conformations independently of hydrolysis and the differences of free energy involved are small compared with the work performed in the power stroke. Only a small degree of thermodynamic coupling is required to ensure that, in the presence of ATP and at physiological temperature, cross-bridges are almost entirely in the 'up' conformation. This is presumably of significance for the efficient operation of the cross-bridge cycle.

We thank Werner Jahn for his help and advice throughout this work, Björn Kindler for providing the program Akkuprog, Gisela Helmig for preparing proteins, Dietmar Manstein for supplying the Dictyostelium myosin motor domain, and Ken Holmes, Jon Kull and Mike Geeves for helpful discussions.

\section{REFERENCES}

1 Geeves, M. A. and Holmes, K. C. (1999) Structural mechanism of muscle contraction. Annu. Rev. Biochem. 68, 687-728

2 Houdusse, A., Szent-Györgyi, A. G. and Cohen, C. (2000) Three conformational states of scallop myosin S1. Proc. Natl. Acad. Sci. U.S.A. 97, 11238-11243

3 Holmes, K. C. (1996) Muscle proteins - their actions and interactions. Curr. Opin. Struct. Biol. 6, 781-789

4 Bagshaw, C. R. and Trentham, D. R. (1974) The characterization of myosin-product complexes and of product-release steps during the magnesium ion-dependent adenosine triphosphatase reaction. Biochem. J. 141, 331-349

5 Sleep, J. A., Trybus, K. M., Johnson, K. A. and Taylor, E. W. (1981) Kinetic studies of normal and modified heavy meromyosin and subfragment-1. J. Muscle Res. Cell Motil. 2, 373-399

6 Millar, N. and Geeves, M. A. (1988) Protein fluorescence changes associated with ATP and adenosine $5^{\prime}$-[ $\gamma$-thio]triphosphate binding to skeletal muscle myosin subfragment 1 and actomyosin subfragment 1. Biochem J. 249, 735-743

7 Rosenfeld, S. S. and Taylor, E. W. (1984) Reactions of $1-N^{6}$-ethenoadenosine nucleotides with myosin subfragment 1 and acto-subfragment 1 of skeletal and smooth muscle. J. Biol. Chem. 259, 11920-11929

8 Trybus, K. and Taylor, E. W. (1982) Transient kinetics of adenosine $5^{\prime}$-diphosphate and adenosine $5^{\prime}$-( $\beta, \gamma$-imido)triphosphate binding to subfragment 1 and actosubfragment 1. Biochemistry 21, 1284-1294

9 Taylor, E. W. (1977) Transient phase of adenosine triphosphate hydrolysis by myosin, heavy meromyosin, and subfragment 1 . Biochemistry 16, 732-739

10 Jahn, W., Urbanke, C. and Wray, J. (1999) Fluorescence temperature-jump studies of myosin S1. J. Anat. 194, 601
11 Jahn, W., Urbanke, C. and Wray, J. S. (1999) Fluorescence temperature jump studies of myosin S1 structure. Biophys. J. 76, 146a

12 Margossian, S. S. and Lowey, S. (1973) Substructure of the myosin molecule. 3. Preparation of single-headed derivatives of myosin. J. Mol. Biol. 74, 301-311

13 Furch, M., Geeves, M. A and Manstein, D. J. (1998) Modulation of actin affinity and actomyosin adenosine triphosphatase by charge changes in the myosin motor domain. Biochemistry 37, 6317-6326

14 Bernasconi, C. F. (1976) Relaxation Kinetics, pp. 201-202, Academic Press, New York

15 Kindler, B. (1997) Ph.D. Thesis, Hannover, Germany

16 Schrumpf, M. and Wray, J. (1992) Structural effects of Al-F and Be-F as analogues of $P_{i}$ in skeletal muscle myosin. J. Muscle Res. Cell Motil. 13, 254

17 Lin, S. H. and Cheung, H. C. (1992) The kinetics of a two-state transition of myosin subfragment 1. A temperature-jump relaxation study. FEBS Lett. 304, 184-186

18 Bauer, C. B., Holden, H. M., Thoden, J. B., Smith, R. and Rayment, I. (2000) X-ray structures of the apo and MgATP-bound states of Dictyostelium discoideum myosin motor domain. J. Biol. Chem. 275, 38494-38499

19 Regnier, M., Lee, D. M. and Homsher, E. (1998) ATP analogs and muscle contraction: mechanics and kinetics of nucleoside triphosphate binding and hydrolysis. Biophys. J. 74, 3044-3058

20 Batra, R. and Manstein, D. J. (1999) Functional characterisation of Dictyostelium myosin II with conserved tryptophanyl residue 501 mutated to tyrosine. Biol. Chem. 380, 1017-1023

21 Fisher, A., Smith, C. A., Thoden, J. B., Smith, R., Sutoh, K., Holden, H. M. and Rayment, I. (1995) X-ray structures of the myosin motor domain of Dictyostelium discoideum complexed with MgADP.BeF ${ }_{x}$ and $\mathrm{MgADP}_{\text {AlF }}^{-}$. Biochemistry $\mathbf{3 4}$ 8960-8972

22 Málnási-Csizmadia, A., Woolley, R. J. and Bagshaw, C. R. (2000) Resolution of conformational states of Dictyostelium myosin II motor domain using tryptophan (W501) mutants: implications for the open-closed transition identified by crystallography. Biochemistry 39, 16135-16146

23 Gulick, A. M., Bauer, C. B., Thoden, J. B. and Rayment, I. (1997) X-ray structures of the MgADP, MgATP $\gamma S$ and MgAMPPNP complexes of the Dictyostelium discoideum myosin motor domain. Biochemistry 36, 11619-11628

24 Shih, W., Gryczynski, Z., Lakowicz, J. R. and Spudich, J. A. (2000) A FRET-based sensor reveals large ATP hydrolysis-induced conformational changes and three distinct states of the molecular motor myosin. Cell 102, 683-694

25 Suzuki, Y., Yasunaga, T., Ohkura, R., Wakabayashi, T. and Sutoh, K. (1998) Swing of the lever arm of a myosin motor at the isomerization and phosphate-release steps. Nature (London) 396, 380-383

26 Lymn, R. and Taylor, E. W. (1971) Mechanism of adenosine triphosphate hydrolysis by actomyosin. Biochemistry 10, 4617-4624

27 Wray, J. S. (1987) Structure of relaxed myosin filaments in relation to nucleotide state in vertebrate skeletal muscle. J. Muscle Res. Cell Motil. 8, 62

28 Wray, J. S., Goody, R. S. and Holmes, K. C. (1988) Towards a molecular mechanism of the contractile cycle. Adv. Exp. Med. Biol. 226, 49-59

29 Rapp, G., Schrumpf, M. and Wray, J. S. (1991) Kinetics of the structural change in the myosin filaments of relaxed psoas fibres after a millisecond temperature-jump. Biophys. J. 59, 35a

Received 10 January 2001/8 May 2001; accepted 7 June 2001 